\title{
Web-Based Integrated Service Discovery Using Agent Platform for Pervasive Computing Environments
}

\author{
Kyu Min Lee, Dong-Uk Kim, Kee-Hyun Choi, and Dong-Ryeol Shin \\ School of Information and Communication Engineering, \\ Sungkyunkwan University, \\ 300 Cheoncheon-dong, Jangan-gu, Suwon, Gyeonggi-do 440-746, Korea \\ $\{\mathrm{kmlee}$, tonykim, gyunee, drshin\}@ece.skku.ac.kr
}

\begin{abstract}
Although current service discovery protocols provide the same basic function of service discovery, they differ significantly in architecture, message exchange pattern, expected operating environment and service representation/description. These differences prevent service interoperability among protocols. To solve this problem, we propose a web-based integrated service discovery mechanism, using an agent platform to guarantee scalability of scope of available services without modifying existing service discovery protocols. The proposed web-based integrated service discovery mechanism provides users with a wider selection of services, and convenient search methods.
\end{abstract}

\section{Introduction}

The increase in the number of different services in pervasive computing environments makes people need service discovery protocols, which help them conveniently find the services they want. Representative service discovery protocols have been classified by academia (INS, INS/Twine, SSDS, Splendor) and industrial standardization bodies (Bluetooth SDP, SLP, UPnP, Jini, Salutation ).

Although they provide the same basic function of service discovery, they differ significantly in architecture, message exchange pattern, expected operating environment and service representation/description. These differences prevent service interoperability among protocols.

To solve this problem, several approaches [1] [2] [3] that support interoperability among service discovery protocols, have been proposed. These approaches, however, have limitations such as translation loss, high maintenance cost, high management cost of service information, the complexity of development, and the modification of existing service.

In this paper, we propose an integrated service discovery mechanism based on an agent platform and the Web, to guarantee scalability of scope of available services without modifying existing service discovery protocols.

The remainder of the paper is organized as follows. Section 2 describes our proposed mechanism and system architecture in detail. Section 3 presents the conclusion. 


\section{Proposed Mechanism and System Architecture}

In this section, we explain integrated service discovery mechanism and system architecture in detail.

\subsection{Integrated Service Discovery Mechanism}

Integrated service discovery mechanism provides users with scalability of services, without any modification of existing service discovery protocols. The key design of the mechanism is that service discovery protocols multicast a state of services, such as service registration and deregistration. Each protocol has a well-known IP and port, so an exterior user or agent can detect the state of services if he joins in the IP and port, i.e. 239.255.255.250:1900 on UPnP and 239.255.255.253:2427 on mSLP. Figure 1 shows the integrated service discovery mechanism.

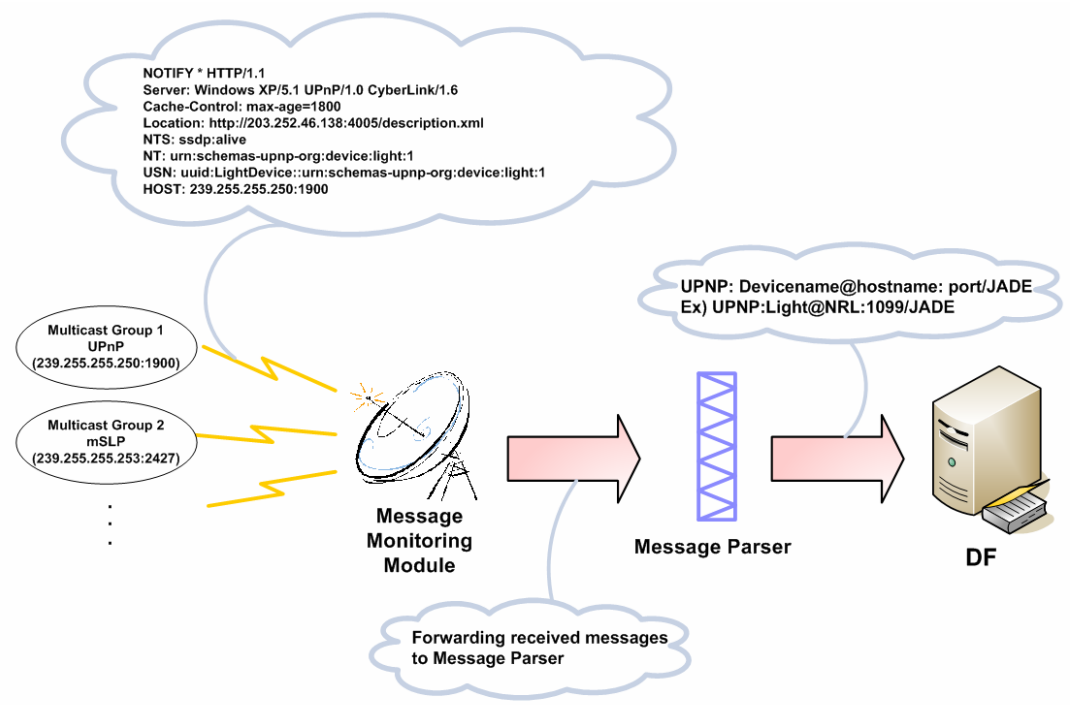

Fig. 1. Integrated Service Discovery

The Message Monitoring module listens to the messages about services from each service discovery protocol. It sends them to the Message Parser. The Message Parser parses them and registers the parsed data to the Directory Facilitator (DF), which is the agent that provides a yellow page service to the agent platform. Finally, agents can freely use the services registered in the DF. In terms of agents, the scope of available services is extended. The following section provides a more detailed explanation of this mechanism. 


\subsection{System Architecture}

Figure 2 shows the proposed system architecture for web-based integrated service discovery using the Java Agent DEvelopment Framework (JADE), which is one of the most popular FIPA-compliant agent platforms.

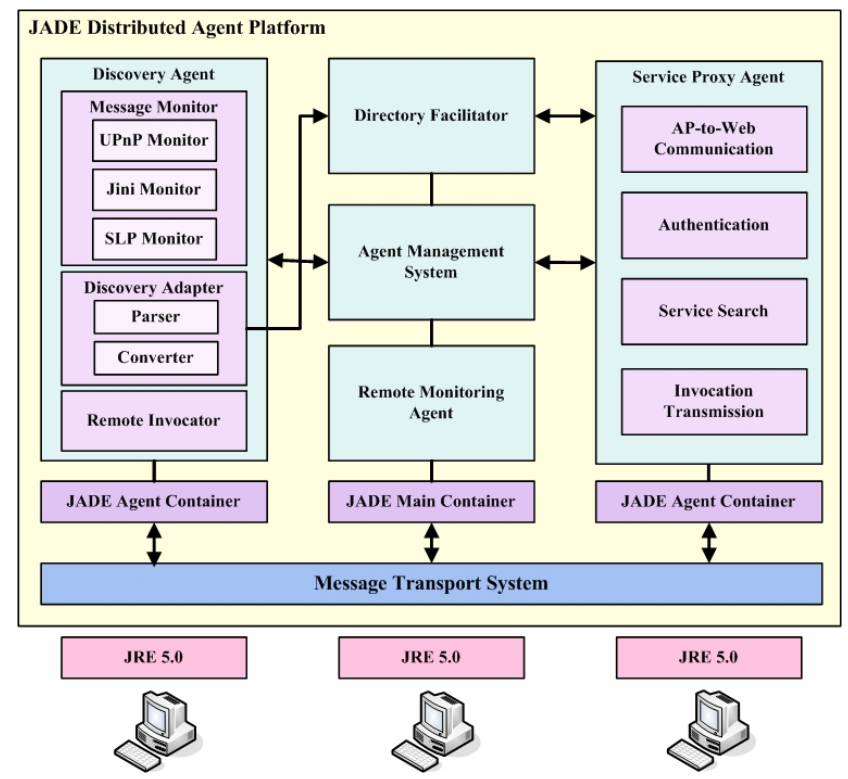

Fig. 2. System Architecture for Web-based Integrated Service discovery

The system consists of Agent Management System (AMS) and DF, which are essential agents of the FIPA-compliant agent platform; RMA, which monitors state of agents; Discovery Agent, and Service Proxy Agent. The Discovery Agent is needed for heterogeneous service discovery. The function of the Service Proxy Agent is to guarantee collaboration with a web server. System processing flow starts in the AP-to-Web Communication module. It sends its host's IP and port number to the web server. When service search from the web server is requested, the Authentication module judges whether a client using the web server has either view authority or invocation authority. The Service Search module then searches available services, and transmits the results to the web server. The Invocation Transmission module sends an invocation message about the service, from the web server requested by the client, to the Remote Invocator module.

Figure 3 depicts the conceptual model of the web-based integrated service discovery system. The architecture is composed of three environments. First, there are domains connected with service discovery protocols such as UPnP, SLP and Jini. Second, there is a JADE environment, as shown in Figure 2, which detects messages of services existing within each service discovery protocol. Finally, there is a web server that supports the viewing of available services and invoking these services. The web 


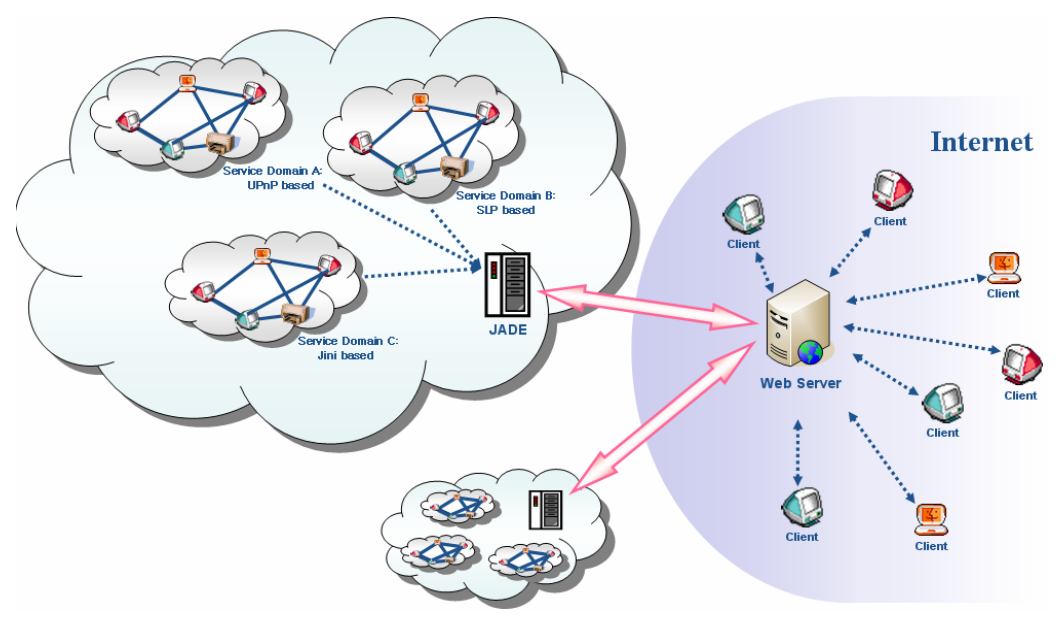

Fig. 3. The Conceptual Model

server requests an available service list to the pre-registered agent platform, according to the clients' request, and then presents the result as a web page.

\section{Conclusion}

This paper mainly contributes to present an integrated service discovery mechanism for heterogeneous services. It is based on an agent platform and the Web, to guarantee scalability of scope of available services, without modifying existing service discovery protocols. The proposed integrated service discovery mechanism provides users with a greater variety of services and convenient search methods.

\section{Acknowledgements}

This research is supported by the ubiquitous Computing and Network (UCN) Project, the Ministry of Information and Communication (MIC) 21 st Century Frontier R\&D Program in Korea.

\section{References}

1. Erik Guttman and James Kempf. Automatic Discovery of Thin Servers: SLP, Jini and the SLP-Jini Bridge. In Proceedings of the 25th Annual Conference of the IEEE Industrial Electronics Society, pages 722-727, 1999.

2. Hiroo Ishikawa et al. A Framework for Connecting Home Computing Middleware. In Proceedings of the IWSAWC2002, 2002.

3. J. Allard, V. Chinta, S. Gundala and G.G Richard. Jini Meets UPnP: An Architecture for Jini/UPnP Interoperability. In Proceedings of the 2003 Symposium on Applications and the Internet, Orlando, 2003. 